\title{
Breaking the Nyquist Barrier : Superresolution Magnetic Imaging from Gradient Data
}

Neil Pendock

Department of Computational and Applied Mathematics, University of Witwatersrand, POBox 197, WITS, 2050 South Africa (under contract to Anglo American's Geophysical Services Department)

De Beers' uses a four sensor magnetic gradiometer system for mineral exploration. Longitudinal, transverse and vertical gradients of the Earth's magnetic field are computed from total field measurements collected by sensors on the tail and wings of a survey aircraft. A superresolution reconstruction of the Earth's total magnetic field is possible since we measure the gradients of the field at slightly different spatial locations.

Two total field data sets may be estimated by integrating the vertical gradient and the longitudinal and transverse gradients. Figure 1 shows the total field at Aredor, Guinea estimated from measured vertical gradient data. Figure 2 shows the total field estimated from the measured longitudinal and transverse gradients. A superresolution total field image is a weighted linear combination of these two data sets.

The two data sets are similar, but not the same. There are several reasons for this :

- When an aircraft flight line in the $\mathrm{x}$ or $\mathrm{y}$ direction intersects a body with magnetization parallel to the direction of the flight line, only one of the $\mathrm{x}$ and $\mathrm{y}$ gradients is nonzero. The $\mathrm{x}$ and $\mathrm{y}$ gradients are independent and may be computed from the $\mathrm{z}$ gradient using the Hilbert transform. The computed gradients will be the same as the measured gradients. In the case of a body with magnetization at some angle $\theta$ to the flight line, both $\mathrm{x}$ and $\mathrm{y}$ gradients are nonzero and not independent. The $\mathrm{x}$ and $\mathrm{y}$ gradients computed from the $\mathrm{z}$ gradient will not be the same. This information may be used to estimate the direction of magnetization of sources.

- The gradients are measured with different sensors having different noise characteristics.

- The gradients are measured at slightly different spatial locations (wingtips and tail). 


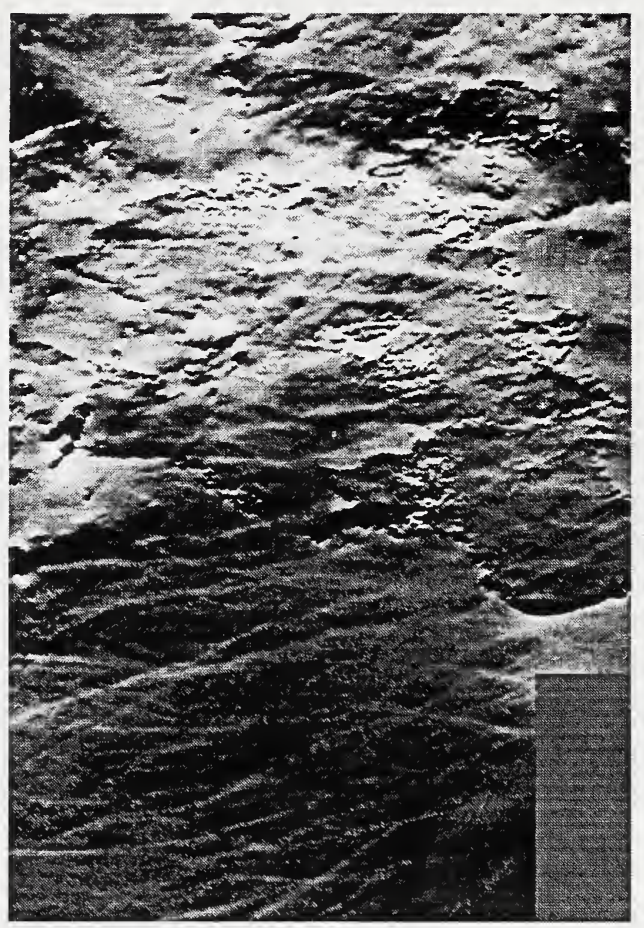

Figure 1 : Total magnetic field integrated from $\mathrm{dz}$

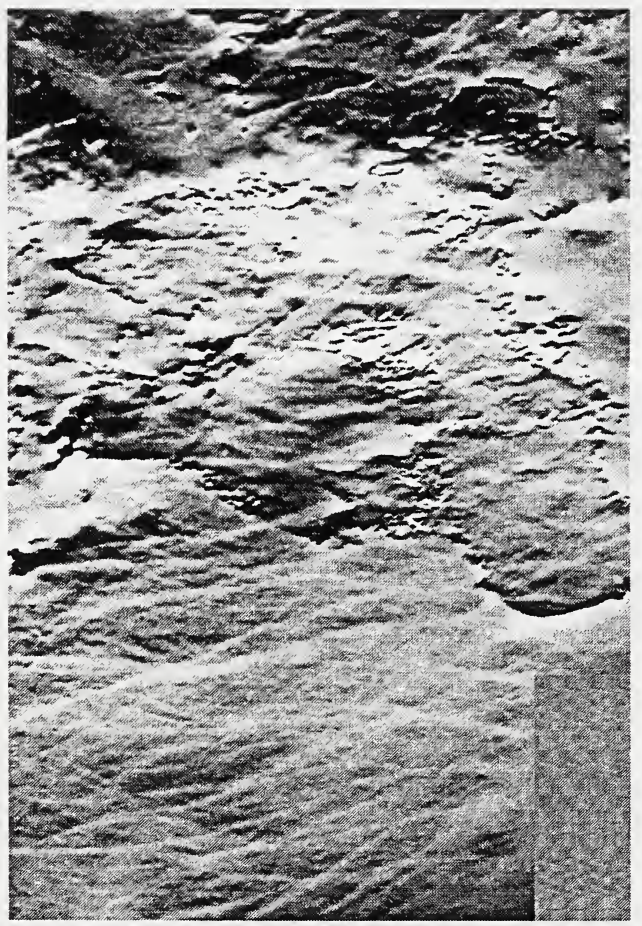

Figure 2 : Total magnetic field integrated from $d x$ and $d y$ 
The effect of source/flight line orientation can be clearly seen by considering an anomaly along an individual profile - the top trace represents the measured vertical magnetic gradient; the bottom trace depicts $\mathrm{dz}$ computed from $\mathrm{dy}$ and $\mathrm{dx}$.

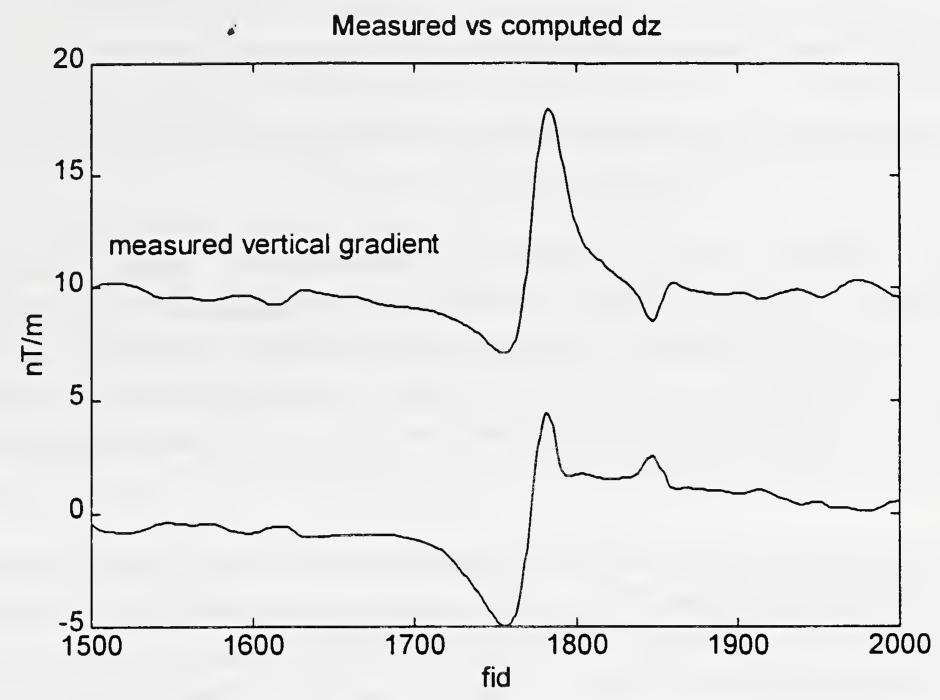

The angle of a two dimensional anomaly source relative to the flight line direction is $\theta=\arctan (-$ $\mathrm{dy} / \mathrm{dx})$. $\theta$ provides information about the orientation of sources.

The two slightly different views of the magnetic field may be combined into a single data set with several benefits over any single view :

increased spatial resolution

reduced noise

enhancement of sources with magnetization at an angle to the flight lines.

We demonstrate the capability of a gradient system to produce total field images with greater spatial resolution, less noise and increased sensitivity than single sensor systems on several magnetic surveys. 